\title{
Des Idées pour observer
}

Ideas for observing

\section{François Sigaut}

\section{(2) OpenEdition}

Journals

Édition électronique

URL : https://journals.openedition.org/tc/4991

DOI : $10.4000 /$ tc. 4991

ISSN : 1952-420X

\section{Éditeur}

Éditions de l'EHESS

\section{Édition imprimée}

Date de publication : 30 juin 2010

Pagination : 84-86

ISSN : 0248-6016

\section{Référence électronique}

François Sigaut, « Des Idées pour observer», Techniques \& Culture [En ligne], 54-55 | 2010, mis en ligne le 30 janvier 2013, consulté le 29 septembre 2022. URL : http://journals.openedition.org/tc/4991

DOI : https://doi.org/10.4000/tc.4991 


\section{DES IDÉES POUR OBSERVER}

in Techniques et culture 10, 1987: 1-12

Le numéro 10 de Techniques et Culture porte sur le même thème que le numéro précédent [Des Idées pour observer], et les articles qui y sont réunis font partie du même ensemble. Leurs auteurs viennent des branches les plus diverses des sciences humaines. Mais on trouvera entre eux, me semble-t-il, sur le plan des concepts et des méthodes, des convergences qui permettent d'espérer qu'en l'occurrence, l'interdisciplinarité n'aura pas été un exercice aussi vain que d'habitude. Nous voyons avec notre cerveau autant qu'avec nos yeux : pour des raisons que j’ai évoquées dans la présentation du numéro précédent, je crois qu’en technologie, le besoin de nouvelles idées pour observer est devenu aujourd'hui particulièrement pressant, si nous voulons continuer à progresser. Et je crois que c'est dans de telles convergences, entre approches indépendantes et différentes au départ, que ces idées nouvelles pourront émerger.

Je voudrais revenir ici sur deux de ces convergences qui me paraissent se dessiner de façon encourageante dans les articles qui composent les numéros 9 et 10 de Techniques et Culture. L'une dans les approches du fait technique par l'objet, et l'autre dans les approches en termes de processus.

\section{Les approches par l'objet : forme, fonctionnement, fonction}

Comme toute formule, celle de « forme, fonctionnement, fonction » n'est certainement pas sans inconvénients, et peut-être faudra-t-il un jour l'abandonner. Pas avant d'en avoir 
tiré les enseignements, toutefois. Or elle nous rappelle qu'il y a toujours plusieurs plans dans la description d'un objet technique, et que c'est la cohérence entre tous ces plans qui véritablement identifie l'objet. Comme l'écrit avec bonheur M. Akrich dans le numéro 9 :

«L'objet technique ne peut pas plus être confondu avec un dispositif matériel qu’avec l'ensemble des usages remplis par ce dispositif : il se définit très exactement comme le rapport construit entre ces deux termes ».

S'agissant d'objets (outils, appareils, etc.), il y aurait au moins trois termes plutôt que deux, mais peu importe pour l'instant. Car c'est bien en trouvant ce rapport construit entre des termes différents, qu'il a appelés forme, fonctionnement et fonction, que J. Cazenobe a pu montrer dans un travail antérieur (1985) : 1) que la question de savoir qui a inventé l'électroaimant avait un sens, et 2) qu'il était possible d'y répondre. L'électroaimant n’apparaît, ni avec la découverte de son "principe» physique (l'aimantation temporaire du fer doux par un courant électrique), ni avec tel ou tel montage destiné à analyser le phénomène. L'électroaimant apparaît lorsqu'est réalisé un dispositif matériel (forme) tirant le meilleur parti possible de l'effet physique en question (fonctionnement) pour permettre pratiquement la commande à distance d'un mouvement (fonction). Lorsqu'on a en vue la cohérence entre ces trois termes, le problème de la distinction entre l'électroaimant et tous les montages expérimentaux qui l'ont précédé se résout de lui-même, et du même coup celui de savoir qui est l'inventeur. Celui-ci s'est servi des travaux de ses devanciers, bien sûr. Mais leurs travaux n'anticipaient pas son invention, parce qu'ils n'en avaient pas le projet. Pour qu'il y ait invention, il faut qu'il y ait un objet nouveau, fonctionnant d'une certaine façon, et conçu pour servir à certaines fins. Tant que la cohérence entre ces trois conditions n'est pas matériellement réalisée, il n'y a pas véritablement invention.

Il se trouve qu'une thématique toute différente m’avait amené à faire cette même distinction entre forme (j'avais parlé d'abord de structure), fonctionnement et fonction depuis plusieurs années (Sigaut 1980). Il n'y a guère de rapports entre l'outillage agricole ancien et l'électroaimant. Et pourtant, les problèmes d'identification de l'objet s'y posent exactement de la même façon, comme on va pouvoir en juger sur l'exemple ultra-classique de la charrue.

On se souvient de la controverse entre P. Leser, auteur en 1931 de Entstehung und Verbreitung des Pfluges, et les deux auteurs de L'Homme et la charrue, A.-G. Haudricourt et M. Jean-Brunhes Delamarre (1955). Leser avait voulu étudier la charrue comme objet historique à l'état pur, si je puis dire. Ce qui impliquait que les caractères directement fonctionnels fussent le plus possible éliminés, au profit de caractères « inexplicables » pour ainsi dire, considérés comme seuls indices fiables de la tradition historique. Pour l'essentiel, ces caractères « inexplicables » étaient des aspects de pure forme - la géométrie triangulaire ou quadrangulaire du bâti de l'instrument, par exemple - sur lesquels il établit logiquement sa classification.

À ce point de vue qu'ils qualifièrent de « formaliste», les auteurs de L'homme et la charrue opposèrent leur point de vue «fonctionnel », selon lequel c'est en étudiant l'instrument au travail qu'on devait trouver l'explication de toutes les différences de formes (1955 : 20-24). Ce qui les conduisit d'abord à la distinction fondamentale entre araire et charrue, ensuite à introduire dans leurs hypothèses des considérations sur les techniques de fabrication (« des bois ployés aux bois rectilignes $»$ ), sur les attelages et les modes de traction, sur la conduite de l'instrument, sur les caractéristiques des sols, etc.

Il n'est pas douteux que ce point de vue «fonctionnel » s'est avéré incomparablement plus fécond que le point de vue «formaliste». Mais avec un recul de plus de trente 
années, nous pouvons voir aujourd'hui cette controverse sous une lumière différente. Le point de vue de Leser n'était pas illégitime. En cherchant à éliminer tout ce qui pouvait s'expliquer par des adaptations fonctionnelles directes, il procédait, au fond, comme les biologistes, qui s'efforcent de distinguer les homologies - indicatrices d'un lien généalogique entre espèces - des convergences - qui ne traduisent que des adaptations à des environnements semblables. Et une douzaine d'années après la parution du livre de Leser, A. Leroi-Gourhan ne fera pas autre chose lorsqu'il proposera de distinguer entre une « tendance », correspondant à une fonction générale et donc sans valeur historique, et des «faits » arrangés par degrés successifs suivant qu'ils sont supposés de moins en moins fonctionnels et de plus en plus spécifiques d'une tradition donnée ${ }^{1}$.

Comme critique d'une histoire trop spéculative, cette démarche représentait assurément un progrès. Et le fait qu'elle fût inspirée de la biologie incite à la prendre au sérieux. L'ennui, c'est qu'une démarche peut être valide en biologie sans nécessairement l'être en anthropologie. Il n'est déjà pas facile, en biologie, de discerner ce qui est fonctionnel de ce qui ne l'est pas. En anthropologie, je me demande si la question a un sens. Et surtout, il y a en biologie une réalité qui rend compte de la présence du non-fonctionnel : l'hérédité, et son support matériel le génome. Mais à quoi donc renvoie le non-fonctionnel, s'il existe, en anthropologie ? À une " tradition » ? Mais qu'est-ce qu'une tradition réduite à n'être que collection de résidus inutiles? Pourquoi serait-elle conservée? Par quelles forces sociales? Ou devons-nous la considérer comme l'expression d'une mystérieuse "personnalité » ethnique, dotée par essence de la propriété de se perpétuer intacte en dépit de toutes les adaptations contingentes? Et à la fin des fins, pourquoi donc ce qui est fonctionnel dans une société aurait-il moins de signification historique que ce qui ne l'est pas?

Mais revenons à nos charrues. Qu'est-ce exactement qu'étudier l'instrument au travail ? Quelles observations cela implique-t-il ? À quelles questions s'agit-il de répondre?

Ce n'est pas faire injure aux auteurs de L'Homme et la charrue, je crois, que d'observer que leur point de vue « fonctionnel » prend davantage en compte le fonctionnement des instruments que leur fonction. Car qu'est-ce qui identifie le travail de l'instrument ? Est-ce l'ensemble des conditions de sol, de climat, le mode de traction, etc. ? Assurément. Mais c'est aussi bien autre chose. Labourer n'a pas de sens précis dans des agricultures où la préparation des blés, des orges, des avoines, exigeait dans le cours de la rotation ordinaire six à huit opérations de labour différentes, chacune ayant ses modalités propres et désignée par un nom propre. Seuls des citadins pouvaient se contenter de dire que les paysans labouraient. Les paysans, eux, étaient occupés à lever les jachères, à biner, à rebiner, à labourer à demeure, à entr'hiverner, à faire les avoines... Et il n'était pas rare que des instruments différents fussent utilisés pour ces différentes opérations. Si bien qu'aujourd'hui, pour comprendre la diversité de ces instruments, nous devons bien sûr les étudier au travail. Mais nous devons surtout déterminer ce qu'était ce travail, en quel point précis du cycle cultural il se situait, c'est-à-dire les résultats qu'on en attendait, les fonctions qu'il s'agissait de remplir².

Ce dont il s'agit, en somme, c'est de reconnaître que dans l'approche par l'objet, il y a plusieurs niveaux de description et d'analyse, tous nécessaires, et qu'il ne faut ni ignorer ni confondre. Les notions de forme, de fonctionnement et de fonction sont simples, on peut même dire intuitives : elles correspondent, en gros, aux questions qu'est-ce que c'est ? comment ça marche? et à quoi ça sert? Une charrue, c'est un certain assemblage de pièces métalliques montées sur un bâti ; ça marche en soulevant ou retournant la terre d'une certaine façon ; et ça sert à exécuter certaines opérations précises du cycle cultural, lequel 


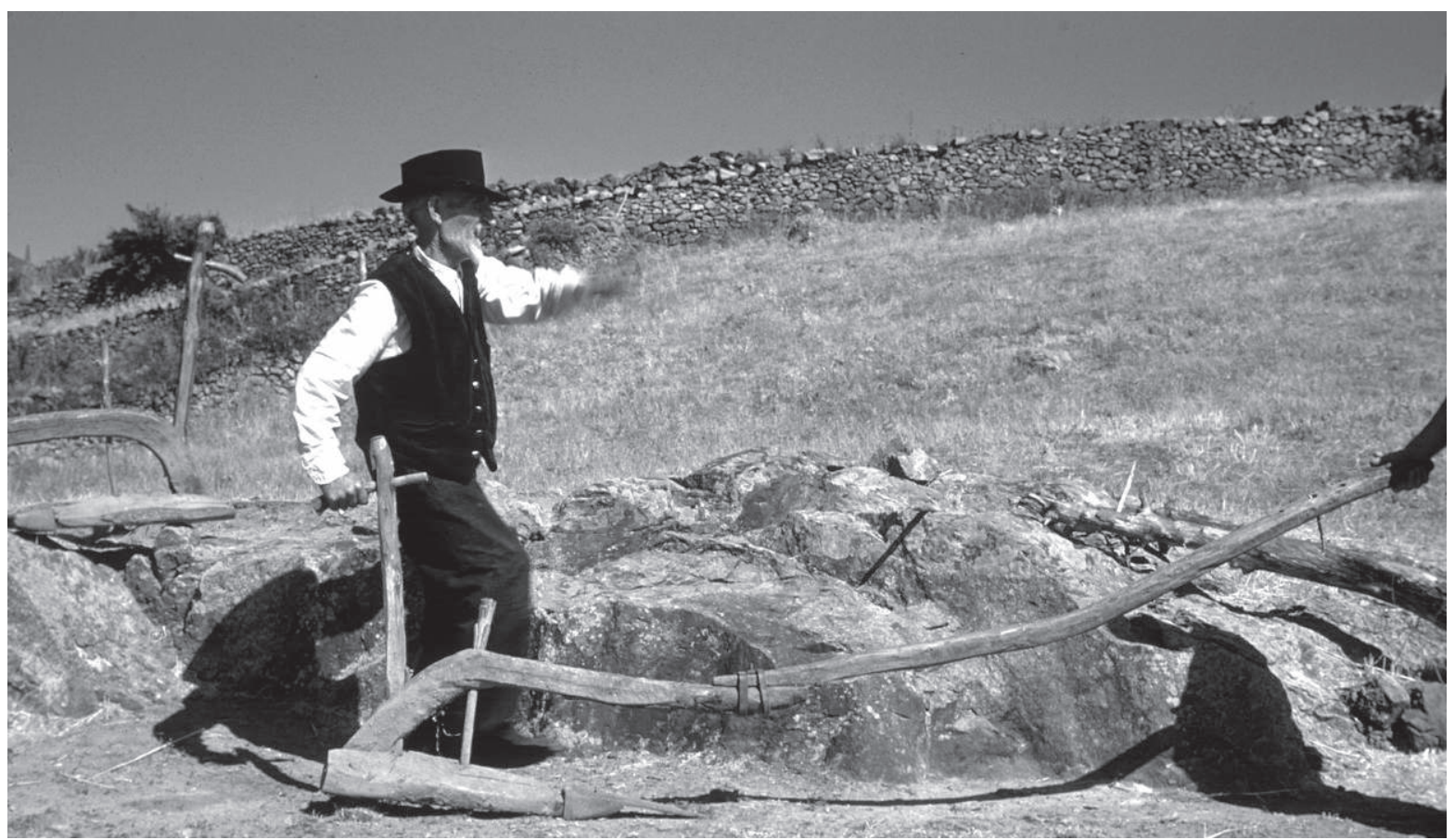

${ }^{\circ}$ Archives I. Chiva

Araire

« Moltifao, 15 Août 1956, M. Ange-François Moltérini présente son araire en position de labours ».

Chiva, I. 2008 (In Techniques \& Culture 50 « Les Natures de I'homme »)

fait lui-même partie d'un certain processus socio-économique de production. On peut certes subdiviser chacune de ces questions, on le doit même sans doute. J.-C. Gardin, cité par O. Buchsenschutz, ne propose pas moins de trois rubriques dans la description de la forme : physique, géométrie, et sémiotique. J. David insiste pour distinguer, dans les fonctions, les usages et la destination : un tournevis est destiné à tourner des vis, mais il a d'autres usages, dont celui universel d'ouvrir les pots de peinture (sauf s'il s'agit d'un tournevis cruciforme); il rejoint en cela une remarque faite depuis longtemps par Radcliffe-Brown qui, déplorant le flou de la notion de fonction, avait proposé de lui substituer des termes comme «emploi », « but » ou «sens » $(1968$ : 287). Quant au fonctionnement, il comprend au moins deux sortes d'aspects : ceux qui concernent l'outil lui-même, et ceux qui concernent la matière travaillée par l'outil. Dans le cas d'une charrue par exemple, ce seront d'une part la traction, la conduite et le réglage, et d'autre part les déplacements de terre déterminés par les pièces travaillantes, les modifications de structure qui en résultent...

Peut-être en fin de compte, J. -F. Quilici-Pacaud (1987) a-t-il raison, et peut-être vaudrait-il mieux parler de niveaux « organique, fonctionnel et relationnel » plutôt que de forme, fonctionnement et fonction. Mais peu importe, au point où nous en sommes. Une discussion approfondie sur tout cela est nécessaire, et il ne faut surtout pas préjuger de ses résultats. Ce dont il s'agit, c'est d'expliciter les idées qui nous ont paru les plus utiles aux uns et aux autres, dans nos recherches sur les objets techniques, de façon à pouvoir les confronter et en tirer ce qui est d'une validité générale. Le point de vue de l'archéologue, celui du muséologue, celui de l'ethnologue, de l'ingénieur, ne s'excluent pas. Ou plus 
exactement nous ne devons pas tolérer qu'ils paraissent s'exclure. Car ce serait admettre l'existence de contradictions définitives entre des corps de connaissances aussi valides les uns que les autres.

\section{Les approches en termes de processus}

La fonction d'un objet, c'est tout ce par quoi il se rattache aux processus dans lesquels on le fait intervenir : il est clair que ces liens sont multiples, et que la notion de fonction est donc plurielle. C'est en tout cas par elle que les études d'objet s'articulent aux études de processus, ce qui lui confère une importance tout à fait centrale dans le champ de la technologie.

Mais qu'est-ce qu'un processus, et comment l'analyser? Nous avons bien tous plus ou moins intuitivement la notion qu'il s'agit de la succession des étapes suivant lesquelles s'organisent la plupart des activités matérielles : du blé au pain, de l'arbre au lit ou à l'armoire par exemple. Mais dans la pratique, cela ne résout pas toutes sortes de problèmes familiers aux lecteurs de Techniques et Culture. Comment décrire un processus? En quelles étapes le découper, et jusqu'où aller dans le détail de ce découpage? Et surtout, pour quoi faire?

Il me semble que là encore, les différentes contributions réunies dans ces deux numéros présentent des convergences encourageantes, et qu'une discussion devrait s'ouvrir pour en dégager les solutions les plus utiles et les plus commodes dans la pratique. Je voudrais ici contribuer à cette discussion par quelques suggestions.

Il me semble par exemple que toute analyse doit résoudre le problème de ses limites, c'est-à-dire celui du niveau élémentaire qu'elle ne peut dépasser sans changer de caractère. C'est ainsi que la chimie est allée jusqu'aux atomes, qui certes se sont avérés sécables, mais par d'autres méthodes que celles de la chimie. Dans un tout autre domaine, et qu'on soit d'accord avec lui ou non, R. Fox a beaucoup clarifié l'analyse de la parenté en identifiant un groupe élémentaire, un atome de parenté si on peut dire, qui est formé par une femme et ses enfants (1967 : 37-40). Je crois que nous clarifierions et faciliterions beaucoup nos descriptions si nous essayions de faire de même en technologie.

La tâche est-elle si difficile? Je me demande au contraire si elle n'est pas assez simple. Car enfin, toute activité technique consiste à modifier l'état matériel de quelque chose (cette modification pouvant n'être qu'un simple déplacement). Or nos descriptions aboutissent très souvent à des schémas du genre:

$$
\text { État matériel } 1 \text { > -----Activité---- > État matériel } 2
$$

Dans le cas des techniques de fabrication, l'état matériel 1 désigne une ou des matières d'œuvre, l'état matériel 2 des produits, sous-produits et déchets. Et il est bien évident que l'état 1 est lui-même le produit d'activités antérieures (nous aurions dû parler d'état N), l'état 2 fournissant la matière d'œuvre à des activités ultérieures (nous aurions dû parler d'état $\mathrm{N}+\mathrm{l}$ ). N'avons-nous pas là notre « atome » de processus ? Un atome qui certes ne peut pas exister à l'état libre puisqu'il fait nécessairement partie d'un ensemble plus vaste - c'est le cas des atomes de la chimie - et un atome qui n'est pas davantage insécable, mais qui délimite deux champs auxquels s’appliquent deux méthodes différentes d'analyse. 
D'un côté, on cherche à décrire ce qu'on peut appeler des filières, c'est-à-dire des processus d'ensemble du type de ceux que j'ai évoqués plus haut : du blé au pain, du minerai à la marmite, etc. Le résultat se présente toujours sous la forme d'un graphe orienté, parfois linéaire, le plus souvent ramifié ou anastomosé de diverses façons. Chaque filière est identifiée par les états successifs de la matière par lesquels elle passe, et il n'est pas étonnant par conséquent que ce soit dans le génie chimique ou biochimique que ce genre d'analyse soit le plus développé (M. Zitt).

Mais la description en termes de filières ne nous renseigne pas, sauf peut-être dans quelques cas très particuliers, sur les diverses activités qui interviennent, c'est-à-dire sur la façon dont on s'y prend pour réaliser pratiquement chaque passage d'un état $\mathrm{N}$ à l'état $\mathrm{N}+1$ de la matière. Car les points de repère ne sont plus les mêmes. Chaque activité est bien aussi un processus. Mais pour analyser ce processus, les états successifs de la matière ne peuvent plus nous servir, puisque par définition il n'y en a plus que deux, l'un initial et l'autre final. Nous avons franchi les limites de notre premier champ d'analyse, et il nous faut donc changer de méthode. Ce qu'il s'agit de décrire maintenant, c'est un ensemble de gestes et de mouvements, qu'il ne sera qu'exceptionnellement possible ou utile de représenter par un graphe orienté. Le modèle n'est plus le génie chimique, c'est la mécanique, à commencer par la mécanique du corps humain (J.-F. Quilici).

Ce n'est pas ici le lieu de développer tout cela, mon but n'étant, encore une fois, que d'ouvrir une discussion. Tous les problèmes ne vont assurément pas se trouver résolus ainsi, comme par un coup de baguette magique! Mais si quelques-uns pouvaient l'être, je crois que le jeu en vaudrait bien la chandelle. C'est sur ces perspectives que je voudrais faire quelques dernières remarques.

Je rappellerai d'abord que cette distinction entre niveaux d'analyse n'est pas entièrement nouvelle. Elle a été anticipée voici bientôt trente ans par Haudricourt (1959 : 46-47) dans un travail où, analysant le système de notation des gestes d'un élève de Taylor, F.B. Gilbreth, il s'aperçut que les gestes élémentaires prétendument représentés par les symboles de Gilbreth étaient en réalité, non pas des « unités de mouvement », mais des « unités d'intention » (en l'espèce, des déplacements simples d'objets). Et que ces « unités d'intention » étaient comparables, non pas aux lettres d'une écriture alphabétique qui supposent l'analyse du mot en phonèmes, mais aux idéogrammes qui ne supposent que l'analyse de l'énoncé en mots, en unités de sens. Nous savons tous que les analogies entre linguistique et technologie peuvent être dangereuses. Mais ici, le parallèle paraît bien justifié. Car c'est bien la modification de l'état de la matière qui donne son sens à l'activité technique. Le geste ou l'objet isolés n'ont pas de sens en eux-mêmes, pas plus que le phonème. Ils n'en reçoivent un qu'indirectement, par le fait qu'ils sont nécessaires à telle ou telle activité3.

Il faudra certainement, pour désigner notre " atome de processus », trouver un autre terme qu'activité, qui n'a comme avantage que d'être aussi neutre et peu connoté que possible. Mais je pense qu'il s'agit d'un concept tout à fait central pour la description technologique. L'activité ne fait pas que délimiter deux niveaux différents de l'analyse des processus techniques. C'est aussi par les activités dans lesquelles on les fait intervenir qu'on détermine les fonctions d'un geste ou d'un objet. Et enfin, c'est à l'intérieur d'une activité bien déterminée qu'on peut parler de lignées ou de filiations techniques : cela sera ma dernière remarque.

Soit par exemple une activité simple : essorer du linge lavé et rincé. Suivant les circonstances, on procédera de diverses façons. On peut poser le linge sur un tréteau pour le laisser égoutter, mais on peut aussi le faire tournoyer pour accélérer cet égouttage. Ou 


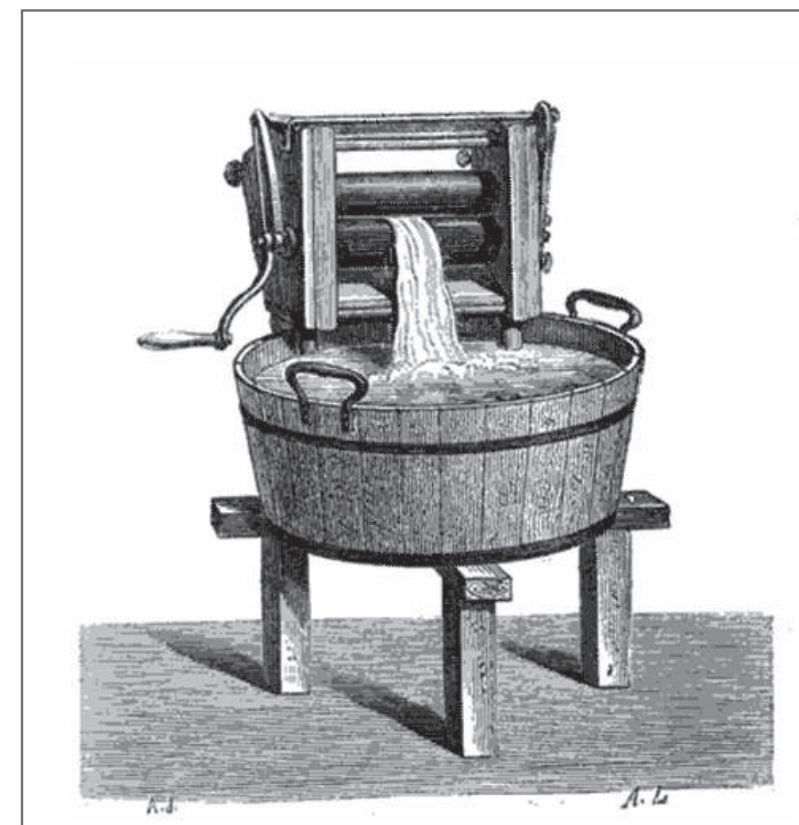

\section{Essoreuse à rouleaux ou calandre Essoreuse centrifuge à tambour}

Nous voyons ainsi se dessiner deux lignées techniques.

La première consiste à soumettre le linge à une compression,

la seconde à le placer dans un champ de force (pesanteur, force centrifuge).

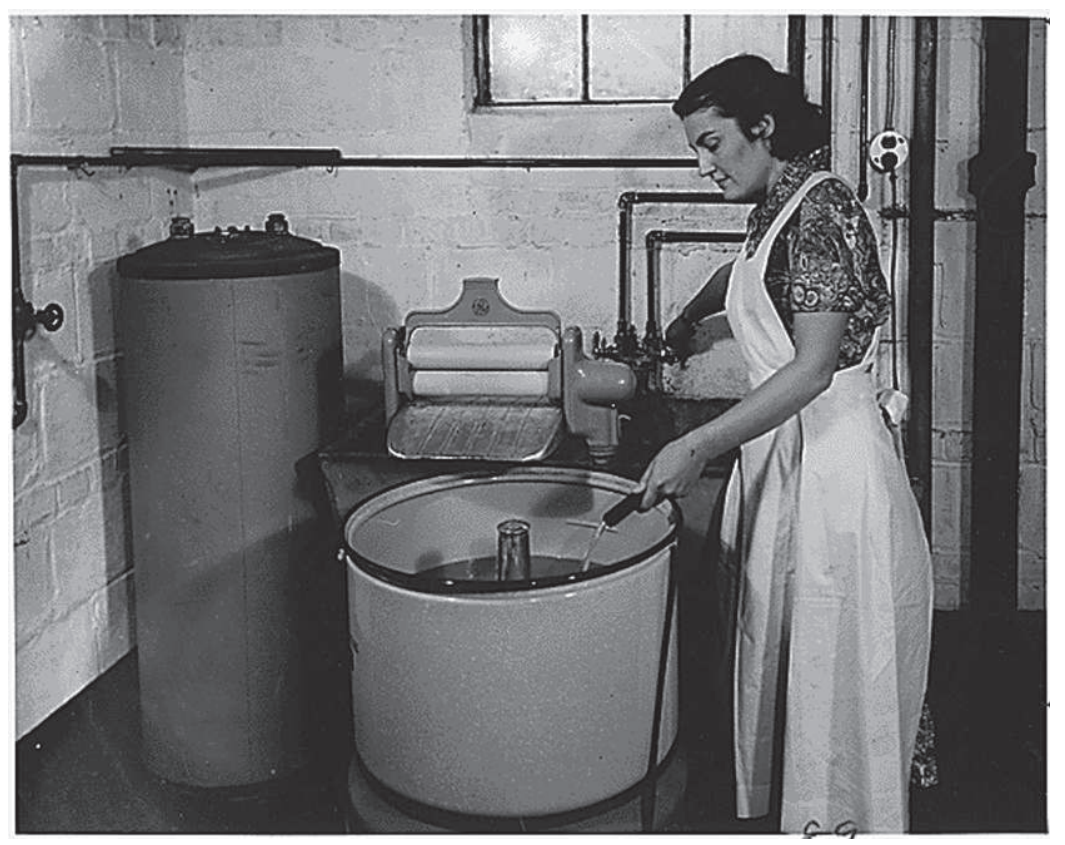

encore on peut presser le linge dans ses mains, le tordre, etc. On voit ainsi se dessiner deux lignées techniques. La première consiste à placer le linge dans un champ de force (pesanteur, force centrifuge), la seconde à le soumettre à une compression. Et très logiquement, l'histoire nous montrerait le développement de machines appartenant aux deux lignées : l'essoreuse centrifuge à tambour, et l'essoreuse à rouleaux ou calandre.

On peut dire, pour reprendre le vocabulaire de Deforge, que ces deux lignées reposent sur deux « principes » différents, qui ont chacun une multitude d'applications en dehors 
de l'essorage du linge. La centrifugation permet d'égoutter la salade, d'écrémer le lait, d'analyser le sang, de séparer les isotopes de l'uranium, de proposer des attractions foraines aux amateurs de sensations fortes, etc. Et l'essoreuse à rouleaux ressemble vraiment beaucoup à l'égreneuse à coton ou au moulin à canne. Mais est-ce le principe qui fait la lignée ? Assurément non, en raison même de cette multitude d'applications qu'on retrouve absolument n'importe où dans l'espace des techniques, si bien que la référence au seul principe n'explique plus rien, ne clarifie plus rien. Il est bien vrai que secouer la salade fait appel au même principe que séparer des isotopes, mais il est vrai aussi que cette seule constatation ne m’apprend pratiquement rien sur la manière de préparer l'uranium fissile, et que je ne risque en l'évoquant aucun ennui avec la D.S.T. !

Ce qui fait une technique, me semble-t-il, c'est l'application d'un principe (ou d'un ensemble de principes) dans une activité, le principe étant identifié en termes mécaniques ou physiques, et l'activité en termes de filières, c'est-à-dire de finalités. Et qu'on ne s'arrête pas à ce vocabulaire, dont je reconnais volontiers qu'il est contestable et inutilement compliqué peut-être ${ }^{4}$. Au lieu de parler de principe et d'activité, il reviendrait au même de parler de fonctionnement et de fonction. Ou plus simplement encore : faire une certaine chose d'une certaine façon, voilà ce qui identifie une technique. Il y a un ordre des fins et un ordre des moyens, qui requièrent chacun des méthodes d'analyse qui leur soient propres. Mais ces analyses restent inachevées et comme suspendues en l'air tant qu'on ne parvient pas à les faire se croiser. Il ne sert à rien de découper un processus en tranches de plus en plus fines si ce découpage ne permet pas de s'interroger sur les différents moyens qui peuvent être mis en œuvre pour le réaliser. Et il ne sert pas à grand chose d'étudier à la loupe une collection d'outils si on n'a pas au moins une vague idée de ce à quoi ils peuvent servir.

Banale cette conclusion? Oh certes! Mais on ne fait pas de technologie sans avoir appris combien le banal est souvent le plus mal connu, parce qu'il est banal précisément. N'est-ce pas pour cette raison exacte qu'on n'a jamais réussi, jusqu'à présent, à proposer une classification des techniques qui soit satisfaisante? Tantôt l'auteur part de la matière, et se voit contraint ensuite d'introduire çà et là des finalités. Tantôt il part au contraire des grandes catégories de fonctions économiques, pour se voir bientôt contraint d'introduire peu à peu des critères plus matériels. Mais toujours empiriquement, sans fil directeur. Je crois qu'avec la notion de lignée comme croisement d'un fonctionnement et d'une fonction, nous avons ce fil directeur, et peut-être la clé de ce qui pourrait être une classification «naturelle» des techniques. Haudricourt plus que quiconque a insisté sur ce qui différencie les classifications artificielles des classifications naturelles, mais c'est une autre de ses idées que je voudrais rappeler pour terminer. « On peut étudier un objet de plusieurs points de vue », écrit-il dans La Technologie, science humaine. " Mais il y a un point de vue plus important que les autres, c'est celui qui peut nous donner les lois de l'apparition et de la transformation de l'objet. » Or nous venons de voir que ce qui produit une lignée technique, c'est le rapport construit entre un fonctionnement et 
une fonction. C'est pourquoi il me semble que la lignée est appelée à jouer en technologie un rôle voisin de celui de l'espèce en biologie. La différence, c'est que l'espèce est une, parce que les êtres vivants s'engendrent les uns les autres. Alors que la lignée technique est irréductiblement duelle, parce que ce sont des hommes qui l'engendrent, chaque fois qu'ils inventent un rapport nouveau entre leurs fins et leurs moyens.

\section{NOTES}

1. Il suffit de relire: 1) l'avertissement méthodologique de P. Leser (1931 : 3 - 50) ou les citations qui en sont faites dans L'homme et la charrue (1955:20-11);2) le passage de L'homme et la matière où sont présentées les notions de tendance et de fait (1943: 27-36); et 3) les articles de S.J. Gould (1986, 1982 : 26-35) sur les aspects historiques de la biologie, pour que le parallélisme des approches saute au yeux. De la part de P. Leser, qui se réclame explicitement de F. Graebner, cela n'a rien d'étonnant; on trouvera l'exposé le plus approfondi de sa position dans un de ses derniers articles (Leser 1977). De la part d'A. Leroi-Gourhan, cela surprendra peut-être. Mais on a l'impression, en le relisant, qu'il a précisément élaboré ses notions de tendance et de fait pour critiquer l'école des Kulturkreise en se plaçant sur le même terrain qu'elle. Si bien qu'aujourd'hui, les ressemblances l'emportent sur les différences. Bien entendu, cette remarque ne porte que sur le texte cité, publié en 1943, pas sur l'œuvre ultérieure d'A. Leroi-Gourhan.

2. Dans le cas des instruments aratoires, la confusion entre fonctionnement et fonction vient sans doute du fait que depuis un siècle au moins, l'agronomie ne prend plus en compte la fonction au sens où on l'entend ici. Le labour y est considéré en soi, comme une catégorie d'opérations, qu'il faut certes raisonner selon le sol, le climat, la saison, le précédent cultural et la culture à venir. Mais il n'y a plus d'intermédiaire entre ce labour « en général », pour ainsi dire, et la multiplicité indéfinie des cas particuliers. Si bien que les instruments ne sont plus définis que par leur fonctionnement -sauf quelques exceptions où la fonction n’a pas pu être tout à fait effacée, comme les charrues décavaillonneuses pour la vigne par exemple. Dans les agricultures anciennes au contraire, il n'y avait pas de labour en général, mais une succession d'opérations dont le programme - nombre de labours, leurs dates et leurs principales caractéristiques - était pour l'essentiel fixé d'avance. Chaque opération avait sa définition propre, si bien que confondre le premier et le deuxième labour de jachère, par exemple, eût été aussi incongru que de confondre le pétrissage de la pâte avec la cuisson du pain (Sigaut 1972, 1977). Dans l'étude des instruments à bras, la diversité des formes et des fonctionnements est beaucoup plus grande, et la notion de fonction prend davantage d'importance (M. Jean-Brunhes Delamarre 1985). Les armes offrent un exemple très semblable d'effacement de la fonction au profit du fonctionnement dans l'historiographie. Dans le cas des armes blanches, la fonction ne peut être définie que dans le cadre d'une escrime donnée, résultant elle-même de tout ce qui fait le caractère habituel des combats (y compris l'absence de combat, pour les armes d'apparat). Dans Milieu et techniques, la dague est l'exemple même de l'arme dont la fonction est bien déterminée - atteindre un adversaire cuirassé en perçant au défaut de la cuirasse dans un combat au corps à corps. Mais quand nous lisons, à propos du sabre oriental qu': « on ne frappe pas avec ce sabre, mais la lame, soit poussée en avant par l'impulsion du cheval ou de la main, soit brusquement ramenée en amère, glisse ou plutôt scie et pénètre de façon à produire d'effroyables blessures » (Bromberger 1981), nous comprenons que cette arme n'a rien de commun avec nos « sabres » d'Occident. La méthode des indices élaborée par A. Leroi-Gourhan (1943 : 59-63, 1945 : 14-21) se situe au niveau du rapport entre forme et fonctionnement. Elle permet de comparer des armes ayant le même fonctionnement, ce dont on ne peut être assuré que quand on en sait assez sur leurs fonctions. Mais elle ne s'applique pas à des armes ayant des fonctionnements différents. C'est une méthode d'analyse, non de classification. Il serait facile de multiplier les exemples. Tous les hameçons, tous les marteaux sont à la fois semblables et différents. Mais un marteau de cordonnier n'est pas un marteau de vitrier ni un marteau d'emballeur. C'est seulement par référence à leurs fonctions, c'est-à-dire à leurs contextes complets d'utilisation, qu'on peut comprendre les détails de leurs fonctionnements, et donc leurs différences de forme. 
3. «Si les chorégraphes et sportifs en sont au balbutiement de l'alphabet, les spécialistes de l'étude du travail industriel en sont encore aux idéogrammes, au stade de l'écriture chinoise », écrit Haudricourt (1959). Je rappelle ici que l'alphabet mécanique de Polhem et les systèmes ou langages symboliques de Babbage et de Reuleaux ont véritablement valeur d'alphabets au sens où Haudricourt l'entend ici, quoiqu'ils ne s'appliquent qu'aux mouvements des organes des machines.

4. Un vocabulaire ne devient d'un maniement commode que quand il est employé par un nombre suffisant de personnes, ce qui, dans les sciences, requiert en général l'adoption de conventions explicites. Toutes les sciences ont leurs congrès de nomenclature et pas seulement la botanique ou la zoologie systématiques. Le vocabulaire que j'emploie dans cet article n'est pas fixé. J'avais pris l'habitude de désigner par «opération » ce que j'appelle ici « activité », mais cela risquait d'introduire des confusions avec l'emploi que fait M. Zitt de ce mot. C'est pour cette raison que j'ai renoncé à parler de « chaîne opératoire », car il me semble nécessaire de déterminer auparavant si cette expression désigne l'enchaînement des activités dans une filière, ou l'enchaînement des gestes dans une activité, deux réalités qu'on doit distinguer avec le plus de netteté possible, comme je crois l'avoir montré. Clarification des concepts et simplification de la nomenclature vont ensemble. Mais l'entreprise suppose un accord général, sinon sur les résultats, au moins sur la nécessité d'y parvenir. En attendant, nous sommes contraints de nous traduire sans cesse les uns les autres pour nous comprendre.

\section{RÉEÉRENCES}

Bromberger, C. 1981 Pourquoi les lames orientales fascinèrent les Occidentaux, Rivages des origines. Marseille: Archives de la Ville (Archives des Cahiers du Sud).

Cazenobe, J. 1985 Essence et naissance de l'électroaimant, History and Technology 2 (2) : 177-201.

Deforge, Y. 1985 Technologie et génétique de l’objet industriel. Paris : Maloine.

Fox, R. 1967 Kinship and marriage. Harmondsworth. Londres : Penguin Books.

Gould, S.J. 1986 Evolution and the triumph of homology, or why history matters, American Scientist 74 (1) : 60-69.

- 1982 Le Pouce du panda. Paris : Grasset.

Haudricourt, A.-G. 1959 Méthode, scientifique et linguistique structurale, L’Année sociologique : 31-48.

Haudricourt, A.-G. \& Jean-Brunhes Delamarre, M. 1955 L'Homme et la charrue. Paris : Gallimard. Jean-Brunhes Delamarre, M. 1985 La Vie agricole et pastorale dans le monde. Paris, Joël Cuénot.

Leroi-Gourhan, A. 1943 L'Homme et la matière. Paris, Albin Michel.

- 1945 Milieu et techniques. Paris, Albin Michel.

Leser, P. 1931 Entstehung und verbreitung des pfluges. Münster : Anthropos.

- 1977 Fritz Graebner. Eine würdigung zum geburtstag am 4. Märs 1977, Anthropos 72(1-2) : 1-55.

Quilici-Pacaud J.-F. Hommage à André leroi-Gourhan. Lettre d'un technicien à ses amis ethnologues, Techniques et culture 10, 45-59.

Radcliffe-Brown, A.R. [1952] 1968 Structure et fonction dans la société primitive. Paris : Éditions de Minuit.

Sigaut, F. 1972 Les Conditions d'apparition de la charrue, Journal d'agriculture tropicale et de botanique appliquée 19 : 10-11 et 442-478.

- 1977 Quelques notions de base en matière de travail du sol dans les anciennes agricultures européennes, Journal d'agriculture tropicale et de botanique appliquée 24 (2-3) : 139-169. 


\section{NOTES ET RÉFÉRENCES DE l'INTRODUCTION}

1. Paillard, J. Réflexions sur l'usage du concept de plasticité en neurobiologie, Journal de Psychologie normale et pathologique, 1976 (1) : 33-47; voir le schéma de la page 37 , repris dans la contribution du même auteur dans J. Piaget \& al. (dir.) 1986, Psychologie. Paris : Gallimard (La Pléiade) p. 1385.

2. Couffignal, L. 1963 La Cybernétique. Paris : PUF. Maunoury, J.-L. 1968 La Genèse des innovations. Paris : PUF. Piaget, J. 1967 Biologie et connaissance Paris, Gallimard. 